\title{
RECENT RESULTS OF THE NUTATION THEORY FOR A RIGID EARTH
}

\section{J. SOUCHAY}

Observatoire de Paris, URA 1125 CNRS, 61, av. de l'observatoire F 75014 Paris, France

\author{
AND \\ H. KINOSHITA \\ Tokyo National Astronomical Observatory \\ Mitaka-shi \\ T181 Tokyo, Japan
}

\begin{abstract}
.
Although the main questions remaining in the frame of the study of the nutation-precession phenomena concern principally the effects due to physical interactions, recent works have shown that even in the simplified case of a rigid Earth model of nutation, important changes have to be taken into account. Here we present the various steps having led to a more complete and more accurate rigid Earth nutation theory, since the pionnier work of Woolard (1953), insisting on the most recent improvements.
\end{abstract}

\section{Introduction}

The most prominent studies concerning the nutation theory try to explain the effects of physical interactions occuring both at the surface of the Earth (ocean loading, tides, atmospheric coupling etc...) or inside it (elasticity, anelasticity, coupling at the core-mantle boundary). Whatever the kind of study involved is, it always refers to a simplified model of nutation, when considering the Earth as a perfectly rigid body. This explains why this model needs to be as accurate as possible. 


\section{The theory of the nutation}

In order to calculate the coefficients of the forced nutation for a rigid Earth model, we need essentially to get suitable analytical developments of the parameters describing the relative motion of the perturbing body (Moon, Sun or planet), that is to say $\lambda, \beta$ and $r$, respectively the longitude, the latitude (with respect to the equinox and the ecliptic) and the distance from the perturbing body to the geocenter.

Then the planetary potential and the luni-solar potential are calculated in function of the three parameters above, and the nutation in longitude and in obliquity are given by the way of the equations of motion. Concerning these last ones, two theories can be used. In Woolard (1953) the equations of motion are given by the theorem of angular momentum, according to which the rate of change of the angular momentum vector is equal to the vector of the resultant torque exerted by the perturbing body. In Kinoshita (1977) the equations of motion are coming from the Hamiltonian of the system of the rotating Earth, after defining 3 angle variables and 3 action variables with respect to the ecliptic and to three fundamental planes: the plane perpendicular to the vector of angular momentum, the plane perpendicular to the axis of figure, and the plane perpendicular to the axis of rotation.

For some reasons, Kinoshita's approach looks more advantageous. At first, a simple change of canonical variables enables one to refer directly to the moving ecliptic of the date, instead of the fixed ecliptic of the basic epoch ( $\mathrm{J} 2000.0)$. Thus the periodical terms of nutation are completely separated from the secular precessional component by using Hori's method (Hori, 1966). At second, the equations giving the coefficients of the nutation for the three axe (axis of angular momentum, axis of figure, axis of rotation) are obtained easily, without using cumbersome Poisson's equations. Last but not least, the calculations related to the interaction between the orbital motion of the Moon and the motion of rotation of the Earth can be carried out in a straightforward manner (Kinoshita and Souchay, 1990).

\section{The recent improvements in rigid Earth nutation}

The tables of nutation given by Kinoshita (1977) included 106 terms larger than 0.1 milliarcsecond (in longitude, obliquity or both). They served as a basis to the determination of the coefficients of the nutation for a non rigid Earth model (Wahr, 1979), which were then adopted by the IAU (Seidelmann, 1980). Vondrak (1983 a,b,c) calculated the influence of the planets on the nutation, which consists in two kinds: the torque exerted on the equatorial bulge of the Earth, and the influence on the relative motion of the Moon and on the Sun, which then causes perturbations on the lunisolar potential. They are respectively called direct and indirect effect. 
In Vondrak's tables, several terms exceed the 0.1 milliarcsecond amplitude and then should have been included in Kinoshita' tables. Moreover, in view of the present VLBI and LLR observations, which reach the 0.01 milliarcsecond of accuracy for individual coefficients, the numerous planetary terms already listed by Vondrak between 0.01 and 0.1 milliarcsecond amplitude can not be neglected anymore.

In order to confirm the analytical determinations of the coefficients of the rigid Earth nutation, one of the best ways was to carry out a numerical integration starting from numerical ephemeris and basic equations. This was done both by Kubo and Fukushima (1988) and Schastok et al. (1988). By comparing the global amount of the nutation with the analytical one given by Kinoshita's tables, they both yield to the conclusion that a big dicrepancy occured both in longitude and obliquity, concerning the $9.3 \mathrm{yr}$ and the $18.6 \mathrm{yr}$ components.

This most important part in this discrepancy originated in the gravitational coupling between the orbital motion of the Moon and the motion of rotation of the Earth. This coupling, not taken into account in Kinoshita (1977), was firstly pointed out by Kubo (1982), who calculated roughly the biggest influence, that is to say the $18.6 \mathrm{yr}$ and the $9.3 \mathrm{yr}$ components, which reached the milliarcsecond. In order to include this coupling and to calculate it very accurately, the two motions above had to be considered altogether, which means that the lunisolar potential, as well as the equations of motion, must be expressed not only in function of the Andoyer canonical variables characterizing the Earth rotation, but also in function of the Delaunay variables characterizing the revolution of the Moon.

The improvement of the theory by considering the effect just mentioned and by including all the coefficients of the nutation at a level significantly smaller than the level of truncation of the IAU 1980 series became urgent, in order to match the accuracy of the determination of the coefficients by the improving observational techniques. This was done by Zhu and Groten (1989) and Kinoshita and Souchay (1990).

A comparison between these two works was studied by Souchay (1993), who showed that the second work was more complete than the first one, noticably because it includes the important direct and indirect effects due to the planets. Nevertheless, the very good agreement between the two works about the calculation of the additional terms due to the spin-orbit coupling effect seems to confirm strongly their values. Notice that the corresponding corrections of Kinoshita and Souchay (1990) for the $18.6 \mathrm{yr}$ and $9.3 \mathrm{yr}$ components are respectively: $-0.43 \sin \Omega+1.19 \sin 2 \Omega$ in longitude, and $0.10 \cos \Omega-0.23 \cos 2 \Omega$ in obliquity, in mas.

In their tables, Kinoshita and Souchay included 277 terms coming from the lunisolar potential, and 119 of planetary origin (direct and indirect). 
Their truncation was set up at 0.005 milliarcsecond. At this level, they had to include coefficients arising from the J3 potential of the Earth, of which the total effect is of the order of 0.1 milliarcsecond peak to peak. An important disagreement with $\mathrm{Zhu}$ and Groten results concerns the $8.85 \mathrm{yr}$ component. The last authors found, in mas: $-0.15 \sin (-l+F+\Omega)$ instead of $-0.11 \sin (-l+F+\Omega)$ for Kinoshita and Souchay, in longitude, and $+0.03 \cos (-l+F+\Omega)$ instead of $-0.09 \cos (-l+F+\Omega)$ in obliquity. Hartmann and Soffel (1994) found results very closed to Kinoshita and Souchay, and in particular concerning this component, although they adopted the same procedure as Zhu and Groten, that is to say a determination of the coefficients of nutation starting from tidal waves.

As it was the case before the improvements of the theory above, new numerical integrations were carried out by Schastok et al. (1989) and Souchay and Kinoshita (1991), which were compared with the nutations given by the new tables of Kinoshita and Souchay (1990). They both showed the drastic decrease of the discrepancy when compared with the previous ones in Schastok et al. (1987) and Kubo and Fukushima (1988). The new discrepancy for the lunisolar contribution does not exceed 0.1 milliarcsecond peak to peak (Souchay and Kinoshita, 1991), both in longitude and in obliquity. This decrease clearly indicates that the various corrections and additioning terms of the analytical results of Kinoshita and Souchay (1990) were justified.

The dicrepancy between numerical integration and analytical results concerning the direct planetary effect does not exceed a few 0.01 milliarcseconds. Very recently, Hartmann and Soffel (1994) computed the nutation of a rigid Earth model due to this effect with the help of the planetary potential development given by Hartmann and Wenzel (1994). They compared their results with Kinoshita and Souchay (1994), and found a very good agreement, the discrepancy still not exceeding a few 0.01 milliarcseconds.

Some new investigations on the motion of precession-nutation were done by Williams (1994), who raised some out-of-phase components due to a tilt of the lunar orbit with respect to the ecliptic plane, caused by the planets. The largest contribution are the $18.6 \mathrm{yr}$ nutations: $-0.030 \sin \Omega+0.137 \cos \Omega$ to $\Delta \psi$ and $-0.028 \sin \Omega+0.003 \cos \Omega$ to $\Delta \varepsilon$ (in mas). This effect should have been added to the tables of Kinoshita and Souchay (1990). Notice that it is the first time that out-of-phase components are included in the rigid Earth model of the nutation. They were usually considered as characterizing exclusively the dissipation in the non-rigid Earth theory.

Another important fact was pointed out by Williams (1994). It concerns the corrections to the obliquity rate due to the direct torque of the planets, to the planetary perturbations on the lunar orbit, and to planetary effects 
through the lunar orbit. Together these corrections are -0.244 mas/yr (Williams, 1994).

The dynamical ellipticity of the Earth $H$ can be determined directly from the value of the general precession in longitude (Kinoshita, 1977). The procedure for the determination is explained in detail in Kinoshita and Souchay (1990). Notice that it does not depend on the Earth model considered (rigid or non-rigid). The value of $H$ given in Kinoshita and Souchay (1990): $H=0.0032739567$, is slightly different from the value in Williams (1994): $H=0.0032737634$, that is to say a relative difference of $6 \times$ $10^{-5}$. This difference is for a very large part explained by the change in the value of the general precession in longitude. Kinoshita and Souchay took the conventional IAU1976 value (Lieske et al., 1977), whereas Williams (1994) adopted a correction of -3.266 mas/yr to this value, which corresponds to very recent VLBI and LLR observations (Herring et al., 1991, Herring, 1991, Mc. Carthy and Luzum, 1991, Steppe et al., 1993, Charlot et al., 1991, Miyamoto and Soma, 1993). The influence of this correction on the main $18.6 \mathrm{yr}$ component of the nutation is about $1 \mathrm{mas}$ in longitude and 0.6 mas in obliquity.

\section{Conclusion}

Since the conventional series of nutation was adopted by the IAU in 1980 , based on the Kinoshita theory of the nutation for a rigid Earth model, two main facts have led to a reconstruction of this last one: firstly, the increasing accuracy of the observational techniques (VLBI,LLR), which enables one to determine coefficients at the level of 0.01 mas. Secondly, the improvement of the rigid Earth theory of the nutation itself. This improvement consists in two parts: one is to keep all the coefficients of nutation above a new truncation level $(0.005$ mas $)$, which means in particular including new contributions due to the planets, to $J_{3}$ etc... The other is to take into account corrections for large coefficients, which are due to a coupling effect between the rotation of the Earth and the revolution of the Moon. Two reconstructions of the nutation for a rigid Earth model were done recently by Zhu and Groten (1989) and Kinoshita and Souchay (1990). For some reasons, explained in detail by Souchay (1993) this last theory looks more accurate and more complete, especially because it includes the planetary effects. In regard to the remarks above, it is recommended that it serves as a basis for any work related to the non rigid Earth nutation.

\section{References}

Charlot, P., Sovers, O.J., Williams, J.G. and Newhall, X.X. (1991), A global VLBI/LLR analysis for the determination of precession and nutation constants. In: Reference 
Systems, IAU Coll. No. 127, eds Hugues J.A., Smith C.A., and Kaplan G.H., United States Naval Observatory, Washington D.C., pp. 228.

Hartmann, T. and Soffel, M. (1994), The nutation of a rigid Earth model: Direct influences of the planets. Astron. J., in press.

Hartmann, T. and Wenzel, H.G. (1994), The harmonic development of the Earth tide generating potential due to the direct effect of the planets. Geophysical Research Letters, submitted.

Herring, T.A. (1991), in: Reference Systems, Proceedings of the 127th. Colloquium of the International Astronomical Union, eds J.A. Hugues, C.A. Smith and G.A. Kaplan.

Herring, T.A., Buffet, B.A., Mathews, P.M. and Shapiro, I.I. (1991), J. Geophys. Res., Vol. 96 No. B5, pp. 8259.

Hori, G.I. (1966), Publ. Astron. Soc. Japan, Vol. 18, pp. 287.

Kinoshita, H. (1977), Celest. Mech., Vol. 15, pp. 277.

Kinoshita, H. and Souchay, J. (1990), Celest. Mech., Vol. 48, pp. 187.

Kubo, Y. (1982), Celest. Mech., Vol. 26, pp. 96.

Kubo, Y. and Fukushima, T. (1988), in: The Earth's Rotation and Reference Frames for Geodesy and Geodynamics, eds A.K. Babcock and G.A. Wilkins, pp. 331.

Lieske J.H., Lederle, T., Fricke, W. and Morando, B. (1977), Astron. Astrophys., Vol. 58, pp. 1.

Mc Carthy, D.D. and Luzum, B.J. (1991), Astron. J., Vol. 102, pp. 1889.

Miyamoto, M. and Soma, M. (1993), Astron. J., Vol. 105, pp. 691.

Schastok, J., Soffel, M. and Ruder, H. (1987), Numerical Solution for the Rotation of the Rigid Earth Model, in: Proc.IUGG symp. U4.

Schastok, J., Soffel, M. and Ruder, H. (1989), Celest. Mech., Vol. 47, pp. 219.

Seidelmann, P.K. (1982), Celest. Mech., Vol. 27, pp. 79.

Souchay, J. (1993), Astron. and Astrophys., 276, pp. 266.

Souchay, J. and Kinoshita, H. (1991), Celest. Mech., Vol. 52, pp. 45.

Steppe, J.A., Oliveau, S.H. and Sovers, O.J. (1993), Earth orientation, reference frames and atmospheric excitation functions. the 1992 IERS Annual Report, IERS technical Note, Vol: 14, ed P.Charlot, pp. R-33.

Vondrak, J. (1983a), Bull. Astr. Inst. Czechosl., Vol. 33, pp. 26.

Vondrak, J. (1983b), Bull. Astr. Inst. Czechosl., Vol. 34, pp. 184

Vondrak, J. (1983c), Bull. Astr. Inst. Czechosl., Vol. 34, pp. 311.

Wahr, J.M. (1979), Ph.D. Thesis, University of Colorado, Boulder, Colorado.

Williams, J.G. (1994), Contributions to the Earth's Obliquity Rate, Precession and Nutation, Astron. J., in press.

Woolard, E.W. (1953), Astronomical Papers for the American Ephemeris and Nautical Almanac XV, Pt. 1, U.S. Government Printing Office, Washington.

Zhu, S.Y. and Groten, E. (1989), Astron. J., Vol. 98, pp. 1104. 